\title{
A Remarkable Patient With Normal Pressure Hydrocephalus: The Importance of Awareness of NPH in Departments Other Than Neurology
}

\author{
Halil Ondera, c, Ibrahim Akkurt ${ }^{b}$
}

\section{To the Editor}

A 62-year-old male with a history of back pain which led to the diagnosis of lumbar disc bulging between L3, L5 was consulted to our department for electrophysiological evaluations to investigate radiculopathy. Following re-interrogation of the complaints of the patient, it was learnt that the patient was admitted to orthopedics and physical medicine and rehabilitation departments multiple times due to walking difficulty and back pain. The patient had begun suffering gait disturbances over the last 3 years and impairment of cognitive functions had been accompanying the clinic. His relative stated a significant urinary incontinency which was in the form of urge urinary incontinence. Neurological examination revealed that the patient was fully orientated and cooperated, but mild apathic. Motor and sensorial examinations were in normal ranges. However, deep tendon reflexes were found to be slightly diminished in lower extremities. The patient's walking was evaluated as "wide based gait" and moderate postural instability was detected considering a cortical malfunction of gait control. When interrogated, the patient and his relatives stated that urinary incontinency was positive and memory and perception disturbances had started over the prior 2 years. Taken together; with a provisional diagnosis of normal pressure hydrocephalus (NPH), cranial magnetic resonance imaging (MRI) was planned which showed enlarged lateral and third ventricles out of proportion to the cortical sulcal enlargement confirming NPH (Fig. 1). Mini mental status examination test was 22 and digit span tests of forward (4)-backward (2) were disturbed. Lumbar puncture provided a significant improvement in gait parameters as well as cognitive status and incontinency (opening pressure was $160 \mathrm{~mm} \mathrm{H}_{2} \mathrm{O}, 30 \mathrm{cc}$ CSF was drained). Hence, after informing the patient and his relatives,

Manuscript submitted August 23, 2017, accepted September 12, 2017

aDepartment of Neurology, Yozgat City Hospital, Ministry of Health, Ankara, Turkey

${ }^{b}$ Department of Neurosurgery, Yozgat City Hospital, Ministry of Health, Ankara, Turkey

${ }^{\mathrm{c} C}$ Corresponding Author: Halil Onder, Department of Neurology, Yozgat City Hospital, Yozgat, Turkey. Email: halilnder@yahoo.com

doi: https://doi.org/10.14740/jnr449w the patient underwent ventriculoperitoneal shunt. On the first month of post-operative evaluation, a dramatic improvement in the patient's locomotion (gait velocity, turning in place, postural instability) was achieved. Significant improvement in his attention and perception according to pre-surgery state was observed during the interview. Forward/backward digit span tests had improved (5/3). In addition, they stated that urinary incontinency was nearly totally recovered.

\section{Discussion}

NPH was firstly described by Adams et al as enlargement of ventricles with normal CSF pressure, and a triad of symptoms: gait disturbance, dementia, and urinary incontinence [1]. As it is a potentially treatable type of dementia, it attracted much interest by a wide circle of physicians, since it was first introduced. By the development of the diagnostic and the therapeutic methods, clinical success rates have been reported to increase to the range of $70-90 \%$ and current guidelines suggest the shunt surgery doubtlessly according to conservative treatment, particularly at early stage of the disease [2]. Nevertheless, even today, only $10-20 \%$ of the NPH patients are known to get the appropriate specialized treatment, while $80 \%$ of patients remain either undiagnosed or misdiagnosed $[2,3]$.

In this report, we present a remarkable patient who had applied multiple times to other departments including orthopedics and physical medicine and rehabilitation due to walking problems. Due to coexisting spinal disk problems, he had undergone to physical exercise programs and given medical treatments of NSAIDs previously. However, the clinic had worsened and he was eventually consulted to neurology department, but for electrophysiological evaluations of possible lumbar radiculopathy. Re-evaluation of the patient in our policlinic revealed rather a cortical gait disorder and other clinical clues of urinary incontinency, cognitive impairment led to a provisional diagnosis of NPH which was confirmed by MRI. Following positive tap test, VP-shunt surgery was conducted which provided a dramatic improvement in all the clinical parameters. For our opinion, this case may represent a crucial report, pointing out that the awareness of this entity should be raised among all physicians considering that clinical suspicion is vital for early diagnosis. Future larger studies to increase the awareness of NPH in departments other than neurology clinics will increase timely neu- 
rology consultations and probably increase the success rate in early diagnosis and management of this critical disease.

\section{Funding}

None.

\section{Conflict of Interest}

None.

\section{References}

1. Adams RD, Fisher CM, Hakim S, Ojemann RG, Sweet WH. Symptomatic Occult Hydrocephalus with "Normal" Cerebrospinal-Fluid Pressure.A Treatable Syndrome. N Engl J Med. 1965;273:117-126.

2. Kiefer M, Unterberg A. The differential diagnosis and treatment of normal-pressure hydrocephalus. Dtsch Arztebl Int. 2012;109(1-2):15-25; quiz 26.

3. Nassar BR, Lippa CF. Idiopathic Normal Pressure Hydrocephalus: A Review for General Practitioners. Gerontol Geriatr Med. 2016;2:2333721416643702.

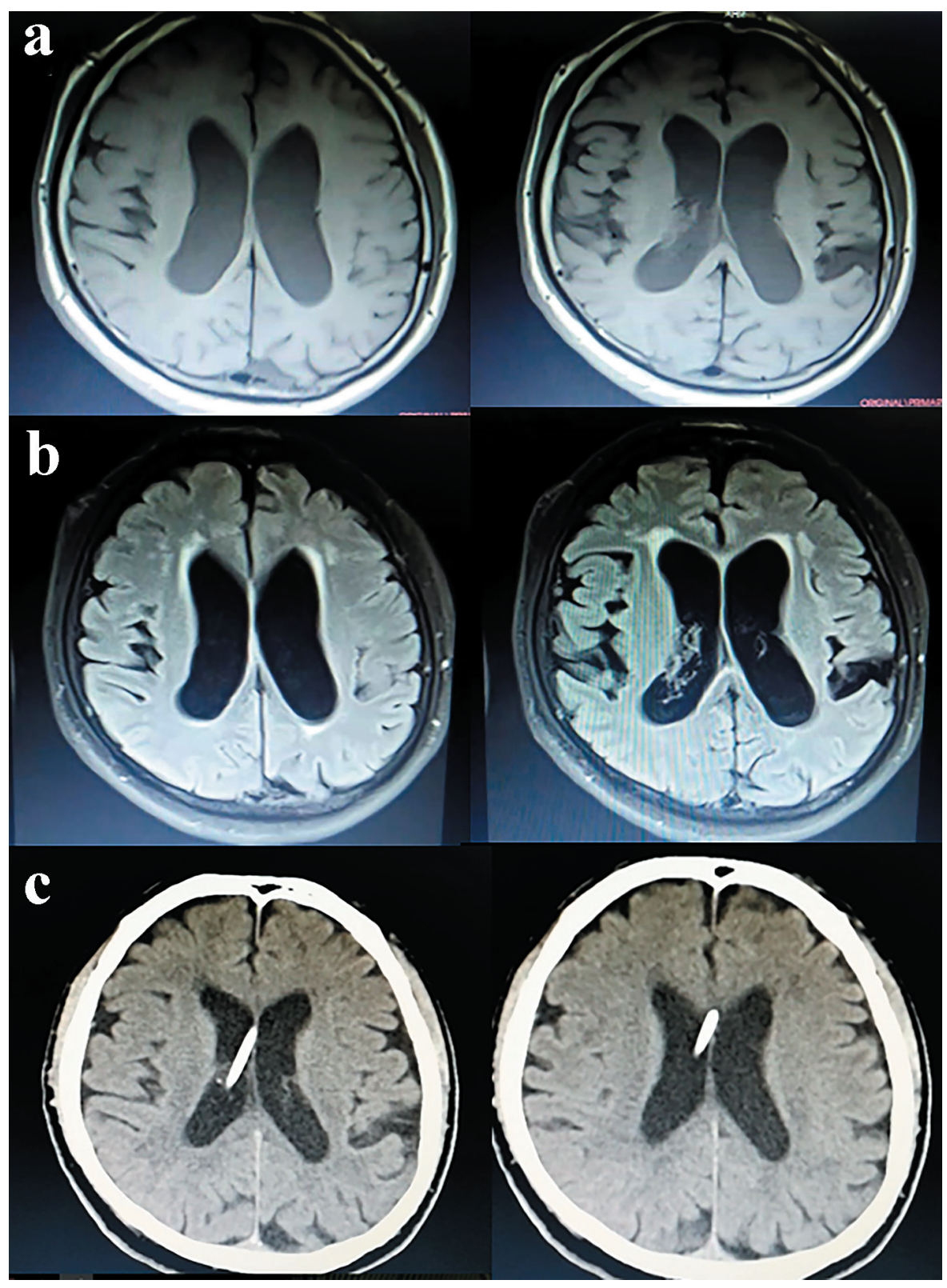

Figure 1. Cranial MRI showing enlarged lateral ventricles out of proportion to the cortical sulcal enlargement (a: T1 sequences; b: FLAIR sequences). (c) Cranial tomography, performed 1 month after the VP-shunt, showing post-operative reduced ventricular volumes. 